\title{
SOME PROPERTIES OF THE EXOTIC MULTIPLICATIONS ON THE THREE-SPHERE
}

\author{
By M. ARKOWITZ and C. R. CURJEL $\dagger$
}

[Received 14 June 1968]

\section{Introduction}

IT is well known that up to homotopy the three-sphere $S^{3}$ admits twelve distinct multiplications or $H$-space structures [see (2), or Lemma 2]. In this note we establish some properties of these different multiplications on $S^{3}$ by elementary group-theoretic arguments.

We work with path-connected topological spaces with base point and homotopy classes of base-point-preserving maps. As usual $[A, B]$ stands for the collection of such homotopy classes of $A$ into $B$. For convenience we do not distinguish notationally between a map and its homotopy class. If $(G, m)$ and $(H, n)$ are two $H$-spaces then a map $f:(G, m) \rightarrow(H, n)$ is called an $H$-map if $n(f \times f)=f m \in[G \times G, H]$. If the $H$-space $(G, m)$ has a two-sided homotopy inverse then a (two-fold) commutator map $\phi: G \times G \rightarrow G$ can be defined by $\phi(x, y)=(x y)\left(x^{-1} y^{-1}\right)$, where the multiplication is denoted by juxtaposition and the homotopy inverse by the exponent ' -1 '. A $k$-fold commutator map $\phi_{k}: G^{k} \rightarrow G$ is then inductively defined by $\phi_{1}=$ the identity map $1, \phi_{2}=\phi$, and

$$
\phi_{k}=\phi\left(\phi_{k-1} \times 1\right) \text {. }
$$

The homotopical nilpotency of $(G, m)$ is said to be equal to $l$ if $\phi_{l+1}$ is nullhomotopic and $\phi_{l}$ is not. We write $\operatorname{nil}(G, m)=l$ for $\phi_{l} \neq 0$ and $\phi_{l+1}=0$, where 0 is the constant map. In the present paper we determine the $B$-maps and the homotopical nilpotency of $S^{3}$ for all possible multiplications.

If $m_{0}$ is the standard multiplication on $S^{3}$ then it is shown in Lemma 2 that the twelve multiplications on $S^{3}$ can be written as

$$
m_{i}=m_{0} \phi^{i} \in\left[S^{3} \times S^{3}, S^{3}\right] .
$$

Here $t=0,1, \ldots, 11, \phi$ denotes the commutator map, and the exponent and juxtaposition are taken with respect to the group structure in $\left[S^{3} \times S^{3}, S^{3}\right]$ induced by $m_{0}$. Finally we denote by $\mathbf{N}$ the map of $S^{3}$ into itself of degree $N$.

+ The first author was supported by a Dartmouth College Faculty Fellowship and the second author partially by the National Science Foundation (NSFGP5874).

Quart. J. Math. Oxdord (2), 20 (1969), $171-6$. 
THEOREM A. The map $\mathrm{N}:\left(S^{3}, m_{r}\right) \rightarrow\left(S^{3}, m_{t}\right)$ of degree $N$ is an $H$-map if and only if $N^{2}(2 t+1) \equiv N(2 r+1) \bmod 24$.

We also prove

THEOREM B. If $r=1,4,7$, or 10 , then $\operatorname{nil}\left(S^{3}, m_{r}\right)=2$. If $r=0,2$, $3,5,6,8,9$, or 11 , then $\operatorname{nil}\left(S^{3}, m_{r}\right)=3$.

In the case of the standard multiplication $(r=t=0)$ Theorem A was proved by us in (1). S. Y. Husseini informs us that he and J. Stasheff have obtained Theorem A by different methods (unpublished). Theorem $B$ was proved by G. J. Porter for $m_{0}$ in (5). Some of the steps in the proof of Theorem B can be found in (3), 175-6 and (4), $\S 10$, where they are established in another way.

Our method of proof consists of examining the group-theoretic properties of $\left[S^{3} \times S^{3}, S^{3}\right]$. With this method it is also possible to retrieve rather easily some known theorems on the multiplications of $S^{3}$ (see Remark 1). A similar discussion of the group $\left[S^{7} \times S^{7}, S^{7}\right]$ leads to results on the multiplications of $S^{7}$. It would be interesting in our opinion to extend our theorems to other $H$-spaces.

\section{Proof of Theorem $A$}

We write $S$ for $S^{3}$ and consider the set $[A, S]$ for any space $A$ as a group, the group operation being induced from the standard multiplication $m_{0}$ of $S$. We write this group multiplicatively even though we denote the identity of the group by 0 . The commutator of two elements $a$ and $b$ is $(a, b)=a b a^{-1} b^{-1}$.

Lemma 1. The group $[S \times S, S]$ is nilpotent of class $\leqslant 2$, and so for any $a, b \in[S \times S, S]$ and integer $n>0$,

$$
(a b)^{n}=(b, a)^{i n(n-1)} a^{n} b^{n} .
$$

Proof. The first assertion is a result of G. W. Whitehead [see (6), Lemma 2.14 and $\S 3$ ], and the second is an identity which is known to hold in a group of nilpotency class $\leqslant 2$.

LeMms 2. The twelve homotopy classes of multiplications on $S$ can be written as $m_{t}=m_{0} \phi^{\prime} \in[S \times S, S], t=0, \ldots, 11$, where $\phi \in[S \times S, S]$ is the commutator map. Moreover, $\phi$ has order 12.

Proof. Consider the cofibre sequence

$$
S \vee S \stackrel{j}{\longrightarrow} S \times S \stackrel{q}{\longrightarrow} S \wedge S \approx S^{6}
$$


ON EXOTIC MULTIPLICATIONS ON THE THREE-SPHERE 173

This gives a long exact sequence

$$
\begin{gathered}
\longrightarrow[\Sigma(S \times S), S] \stackrel{\Sigma j^{*}}{\longrightarrow}[\Sigma(S \vee S), S] \longrightarrow\left[S^{6}, S\right] \\
\stackrel{q^{*}}{\longrightarrow}[S \times S, S] \stackrel{j^{*}}{\longrightarrow}[S \vee S, S]
\end{gathered}
$$

where $\Sigma$ denotes reduced suspension. It is well known and easily proved that $\Sigma j^{*}$ and $j^{*}$ are epimorphisms. Thus we get a short exact sequence of groups and homomorphisms

$$
0 \longrightarrow \pi_{6}(S) \stackrel{q^{*}}{\longrightarrow}[S \times S, S] \stackrel{j^{*}}{\longrightarrow} \pi_{3}(S) \oplus \pi_{8}(S) \longrightarrow 0
$$

A multiplication $m$ on $S$ is an element of $[S \times S, S]$ such that

$$
j^{*}(m)=1 \oplus 1 \text {, }
$$

where $1 \in \pi_{3}(S)$ is the homotopy cless of the identity map. Thus the collection of multiplications is just the coset of $\operatorname{Ker} j^{*}$ which contains $m_{0}$. Hence it suffices to show that $\operatorname{Im} q^{*}$ is a cyclic group of order 12 generated by $\phi$. Now it is known that $\pi_{6}(S)$ is a cyclic group of order 12 generated by $\langle 1,1\rangle$, the Samelson product of $1 \in \pi_{3}(S)$ with itself [see (3) 176]. But by the definition of the Samelson product $q^{*}\langle 1,1\rangle=\phi$. This completes the proof of the lemma.

Proof proper of Theorem $A$. First note that $m_{0}=p_{1} p_{2}$ and $\phi=\left(p_{1}, p_{2}\right)$ in $[S \times S, S]$ where $p_{i} \in[S \times S, S]$ is the projection on to the $i$ th factor. Assume that $N>0$. Then

$$
\begin{aligned}
m_{l} \circ(\mathbf{N} \times \mathbf{N}) & =\left(p_{1} p_{2} \phi^{l}\right) \circ(\mathbf{N} \times \mathbf{N}) \\
& =\left(\mathbf{N} p_{1}\right)\left(\mathbf{N} p_{2}\right)\left(\mathbf{N} p_{1}, \mathbf{N} p_{2}\right)^{l} \\
& =p_{1}^{N} p_{2}^{N}\left(p_{1}, p_{2}\right)^{N^{\ell}}
\end{aligned}
$$

since the commutator is biadditive in a group of nilpotency $\leqslant 2$. On the other hand,

$$
\begin{aligned}
\text { Nom } & =\left(p_{1} p_{2} \phi^{r}\right)^{N} \\
& =\left(\phi^{r}, p_{1} p_{2}\right)^{\dagger N(N-1)}\left(p_{1} p_{2}\right)^{N} \phi^{r N}, \quad \text { by Lemma 1, } \\
& =\left(p_{1} p_{2}\right)^{N} \phi^{r N} \quad \text { since }\left(\phi^{r}, p_{1} p_{2}\right)=0 \\
& =\left(p_{2}, p_{1}\right)^{t N(N-1)} p_{1}^{N} p_{2}^{N} \phi^{r N}, \text { by Lemma 1, } \\
& =p_{1}^{N} p_{2}^{N}\left(p_{1}, p_{2}\right)^{r-1 N(N-1)} .
\end{aligned}
$$

Thus $\mathrm{N}$ is an $H$-map if and only if $\phi^{N^{4}}=\phi^{r N-1 N(N-1)}$. Since $\phi$ has order 12 by Lemma 2, this is the case if and only if

$$
N^{2}(2 t+1) \equiv N(2 r+1) \bmod 24 .
$$

If $N<0$ write $N=(-N)(-1)$ and repeat the above argument. 


\section{Proof of Theorem $B$}

We begin with a simple lemma on homotopy inverses.

Leman 3. The map $-1 \in[S, S]$ of degree -1 is a two-sided identity for every multiplication on $S$.

Proof. A left inverse $v_{t} \in[S, S]$ for $m_{l}$ is characterized by the equation $v_{t} .1=0$ in $[S, S]$, where the dot '. ' is the operation in $[S, S]$ induced by $m_{t}$. But this operation coincides with the usual group operation in the homotopy group $[S, S]=\pi_{3}(S)$. Thus $\nu_{t}=-1$ is the unique solution of the equation. Similarly the right inverse for $m_{t}$ is -1 .

Next we introduce the following notation: $\phi_{k}^{(r)}$ is the $k$-fold commutator map with respect to the multiplication $m_{r}$. It is well known that each $\phi_{k}^{(r)}$ induces a unique homotopy class $\psi_{k}^{(r)}: \wedge^{k} S \rightarrow S$ with $\psi_{k}^{(r)} \circ q_{k}=\phi_{k}^{(r)}$, where $\wedge^{k} S$ is the smashed product $S \wedge \ldots \wedge S(k$ times $) \approx S^{3 k}$ and $q_{k}$ is the projection of $S \times \ldots \times S$ on to $\wedge^{k} S$. For $\alpha \in \pi_{p}(S)$ and $\beta \in \pi_{q}(S)$ denote the Samelson product relative to $m_{r}$ by $\langle\alpha, \beta\rangle_{r} \in \pi_{p+\alpha}(S)$. We write $\phi_{k}$ for $\phi_{k}^{(0)}, \phi^{(r)}$ for $\phi_{2}^{(r)}, \psi_{k}$ for $\psi_{k}^{(0)}, \psi^{(r)}$ for $\psi_{2}^{(r)}$, and $\langle\alpha, \beta\rangle$ for $\langle\alpha, \beta\rangle_{0}$.

LEMMA 4. (a) $\phi^{(r)}=\phi^{2 r+1} \in[S \times S, S]$;

(b) $\psi^{(r)}=\psi^{2 r+1} \in\left[S^{6}, S\right]$

(c) $\langle\alpha, \beta\rangle_{\tau}=(2 r+1)\langle\alpha, \beta\rangle$ for $\alpha \in \pi_{p}(S)$ and $\beta \in \pi_{q}(S)$.

Proof. Clearly $(b)$ implies $(c)$ since $\langle\alpha, \beta\rangle_{r}=\psi^{(r)}(\alpha \wedge \beta)$. Also $(b)$ is an immediate consequence of $(a)$. Thus it suffices to prove $(a)$ : Write '.' for the operation in $[S \times S, S]$ obtained from $m_{r}$ and use juxtaposition for the standard multiplication in $[S \times S, S]$. Then, by Lemma 3 ,

$$
\phi^{(r)}=\left(p_{1} \cdot p_{2}\right) \cdot\left(p_{1}^{-1} \cdot p_{8}^{-1}\right),
$$

where $p_{1}$ and $p_{2}$ are the two projections $S \times S \rightarrow S$ and the exponent ' -1 ' denotes the inverse in $[S \times S, S]$ with respect to the standard multiplication in $[S \times S, S]$. Then

$$
\begin{aligned}
\phi^{(r)} & =p_{1} p_{2}\left(p_{1}, p_{2}\right)^{r} \cdot p_{1}^{-1} p_{2}^{-1}\left(p_{1}^{-1}, p_{2}^{-1}\right)^{r} \\
& =p_{1} p_{2} \phi^{r} p_{1}^{-1} p_{2}^{-1} \phi^{r}\left(p_{1} p_{2} \phi^{r}, p_{1}^{-1} p_{2}^{-1} \phi^{r}\right)^{r} \\
& =\phi^{2 r+1}\left(p_{1} p_{2} \phi^{r}, p_{1}^{-1} p_{2}^{-1} \phi^{r}\right)^{r} .
\end{aligned}
$$

But by repeatedly using the biadditivity of commutators in the group $[S \times S, S]$ of nilpotency class $\leqslant 2$ one easily sees that

$$
\left(p_{1} p_{2} \phi^{r}, p_{1}^{-1} p_{2}^{-1} \phi^{r}\right)=0 .
$$

This completes the proof. 
Proof proper of Theorem $B$. We first note that $\phi_{k}^{(r)}=0$ if and only if $\psi_{k}^{(r)}=0$. Now $\psi_{2}^{(r)}=\psi^{(r)}=\langle 1,1\rangle_{r}=(2 r+1)\langle 1,1\rangle$ for $1 \in \pi_{3}(S)$. But $\langle 1,1\rangle \in \pi_{\theta}(S)$ is an element of order 12. Therefore,

Next,

$$
\psi_{2}^{(r)} \neq 0 \text { for all } r \text {. }
$$

$$
\begin{aligned}
\psi_{s}^{(r)} & =\left\langle\langle 1,1\rangle_{r}, 1\right\rangle_{r} \\
& =(2 r+1)\langle(2 r+1)\langle 1,1\rangle, 1\rangle \\
& =(2 r+1)^{2}\langle\langle 1,1\rangle, 1\rangle .
\end{aligned}
$$

But $\langle\langle 1,1\rangle, 1\rangle \in \pi_{9}\left(S^{3}\right) \approx Z_{3}$ is an element of order 3 [see for instance (2) § 3]. Therefore $\psi_{3}^{(r)}$ (and consequently $\phi_{3}^{(r)}$ ) is trivial if and only if $r=1,4,7$, or 10. Thus $\operatorname{nil}\left(S, m_{r}\right)=2$ for $r=1,4,7$, or 10. Finally note that $\left\langle\left\langle\langle 1,1\rangle_{r}, 1\right\rangle_{r}, 1\right\rangle_{r}$ is a multiple of $\langle\langle\langle 1,1\rangle, 1\rangle, 1\rangle$. To complete the proof of Theorem B it suffices to show that this latter Samelson product is trivial.

LmMar 5. If $1 \in \pi_{3}(S)$ is the homotopy class of the identity map then $\langle\langle\langle\mathbf{1}, \mathbf{1}\rangle, \mathbf{1}\rangle, \mathbf{1}\rangle=0 \in \pi_{12}(S)$.

Proof. Let $\theta=\langle\langle\langle 1,1\rangle, 1\rangle, 1\rangle$ and $\eta=\langle\langle 1,1\rangle,\langle 1,1\rangle\rangle$. First note that $3\langle\langle 1,1\rangle, 1\rangle=0$ by the Jacobi identity and so $3\langle\langle\alpha, \beta\rangle, \gamma\rangle=0$ for any elements $\alpha, \beta, \gamma$. Thus $3 \eta=0$ and $3 \theta=0$. But $\eta=-\eta$ by anticommutativity, and so $2 \eta=0$. Hence it follows that $\eta=0$. Now apply the Jacobi identity to the elements $\langle 1,1\rangle, 1$, and 1 to obtain $2 \theta+\eta=0$. Thus $2 \theta=0$. But we observed earlier that $3 \theta=0$. Therefore $\theta=0$.

Remark 1. It is possible with our methods to retrieve some known theorems on the multiplications of $S$. We can prove that all multiplications on $S$ satisfy the Moufang identity [see (4) $\S 9$ ] and can determine which of them are homotopy-associative [see (2) Theorem 1.3]. These results require an examination of the group $[S \times S \times S, S]$, which is nilpotent of class $\leqslant 3$. For example, $m_{r}$ is homotopy-associative if and only if $q_{1} \cdot\left(q_{2} \cdot q_{3}\right)=\left(q_{1} \cdot q_{2}\right) \cdot q_{3}$ in $[S \times S \times S, S]$, where $q_{i}$ is the projection on to the ith factor. This equation, together with facts on commutators in groups of nilpotency class $\leqslant 3$, reduces to a simple identity in $[S \times S \times S, S]$ in terms of $m_{0}$. We note that the multiplications on $S$ which are homotopy-associative are precisely those which in Theorem $B$ were shown to be of homotopical nilpotency 3.

Remark 2. The definition of homotopical nilpotency for a nonhomotopy-associative $H$-space depended on the bracketing in the definition of a commutator map. It follows from our proof of Theorem B that the homotopical nilpotency with respect to one bracketing is the same as that with respect to any other bracketing of the commutator. 
176 ON EXOTIC MULTIPLICATIONS ON THE THREE-SPHERE

\section{REFERENCES}

1. M. Arkowitz and C. R. Curjel, 'On maps of $B$-spaces', Topology, 6 (1967) $137-48$.

2. I. M. James, 'Multiplications on spheres (II)', Trans. Amer. Math. Soc. 84 (1057) 545-58.

3. ㄴ 'On $B$-spaces and their homotopy groups', Quart. J. Math. (Oxford) (2) $11(1960) 161-78$.

4. C. W. Norman, 'Homotopy loops', Topology, 2 (1963) 23-43.

5. G. J. Porter, 'Homotopical nilpotency of $S^{2}$ ', Proc. Amer. Math. Soc. 15 (1964) 681-2.

6. G. W. Whitehead, 'On mappings into group-like spaces', Oomm. Math. Helv. 28 (1954) 320-8.

Dartmouth College

Hanover, N.H., and

Mathematical Institute

Oxford

University of Washington

Seattle, and

Forschungsinstitut für Mathematik, E.T.H.

Zürich 